\title{
Gluconate 5-dehydrogenase (Ga5DH) participates in Streptococcus suis cell division
}

\author{
Zhongyu Shi ${ }^{1,2}$, Chunling Xuan ${ }^{1,2}$, Huiming Han ${ }^{1}$, Xia Cheng $^{1,3}$, Jundong Wang ${ }^{3}$, Youjun Feng ${ }^{4}$, \\ Swaminath Srinivas ${ }^{5}$, Guangwen $\mathrm{Lu}^{1}$, George F. Gao ${ }^{1,2,6,7 凶}$ \\ ${ }^{1}$ CAS Key Laboratory of Pathogenic Microbiology and Immunology, Institute of Microbiology, Chinese Academy of Sciences, \\ Beijing 100101, China \\ 2 University of Chinese Academy of Sciences, Beijing 100049, China \\ ${ }^{3}$ College of Animal Science and Technology, Shanxi Agricultural University, Taigu 030801, China \\ ${ }^{4}$ Department of Microbiology, University of Illinois, Urbana, IL 61801, USA \\ ${ }^{5}$ Department of Biochemistry, University of Illinois, Urbana, IL 61801, USA \\ ${ }^{6}$ Laboratory of Protein Engineering and Vaccines, Tianjin Institute of Industrial Biotechnology, Chinese Academy of Sciences, \\ Tianjin 300308, China \\ ${ }^{7}$ Research Network of Immunity and Health (RNIH), Beijing Institutes of Life Science, Chinese Academy of Sciences, \\ Beijing 100101, China \\ $凶$ Correspondence: gaof@im.ac.cn (G. F. Gao) \\ Received April 1, 2014 Accepted May 4, 2014
}

\begin{abstract}
Bacterial cell division is strictly regulated in the formation of equal daughter cells. This process is governed by a series of spatial and temporal regulators, and several new factors of interest to the field have recently been identified. Here, we report the requirement of gluconate 5-dehydrogenase (Ga5DH) in cell division of the zoonotic pathogen Streptococcus suis. Ga5DH catalyzes the reversible reduction of 5-ketogluconate to D-gluconate and was localized to the site of cell division. The deletion of Ga5DH in S. suis resulted in a plump morphology with aberrant septa joining the progeny. A significant increase was also observed in cell length. These defects were determined to be the consequence of $\mathrm{Ga} 5 \mathrm{DH}$ deprivation in S. suis causing FtsZ delocalization. In addition, the interaction of FtsZ with Ga5DH in vitro was confirmed by protein interaction assays. These results indicate that Ga5DH may function to prevent the formation of ectopic $Z$ rings during $S$. suis cell division.
\end{abstract}

KEYWORDS Streptococcus suis, Ga5DH, cell shape, cell division, FtsZ localization

\section{INTRODUCTION}

Streptococcus suis (S. suis) is an emerging zoonotic pathogen that causes life-threatening diseases, including septicemia, meningitis, and endocarditis (Kay et al., 1995; Mazokopakis et al., 2005), with more than 750 reported human cases of infection worldwide (Feng et al., 2010). Amongst the 35 known serotypes of S. suis, serotype 2 (SS2) is considered to be the most pathogenic and prevalent form in both pigs and humans (Nghia et al., 2008). In addition to sporadic cases of human SS2 infections (Feng et al., 2009; Feng et al., 2010), two large scale outbreaks of human SS2 endemics, with an unprecedented high rate of morbidity and mortality in China, were reported in 1998 and 2005 (Tang et al., 2006). Potent inhibitors and therapeutic agents to effectively control $S$. suis infection are required to address the re-emergence of SS2 as a zoonotic pathogen in humans and the rapid increase of antibiotic-resistant strains among clinical isolates.

D-gluconate, an important carbon source for many microorganisms, is required for Escherichia coli (E. coli) to colonize the streptomycin-treated mouse large intestine (Sweeney et al., 1996), suggesting that gluconate might play an important role in both bacterial survival and virulence. Membrane-bound gluconate 5-dehydrogenase (Ga5DH) catalyzes the inter-conversion of D-gluconate and 5-keto-Dgluconate, while simultaneously generating NADPH, which acts as a hydrogen donor for many biosynthetic processes. Cell cycle proteins have traditionally been an attractive target for antibacterial agents, but with our increasing understanding in this area, Ga5DH also seems like an effective 
target for the development of novel potent antibacterial agents. In our previous work, S. suis Ga5DH was characterized both structurally and enzymatically (Zhang et al., 2009).

Cell division is initiated by the formation of a cytokinetic ring at the prospective division site (Bi \& Lutkenhaus, 1991), which leads to the production of two identical daughter cells. Ellipsoid-shaped bacteria such as $S$. suis divide at the midpoint of the cell along successive parallel planes perpendicular to the long axis. The first known event in bacterial cytokinesis is the polymerization of the tubulin homologue GTPase FtsZ into a ring structure at the prospective site of division (Bi and Lutkenhaus, 1991). The polymerization of FtsZ into the $Z$ ring at the future division site is critical for cell division because it guides septum synthesis, location, and shape (Addinall \& Lutkenhaus, 1996). Diverse cell division components interact with FtsZ to regulate FtsZ assembly. In E. coli, two division proteins, ZipA and FtsA, directly interact with FtsZ and cooperate in anchoring FtsZ to the membrane (Pichoff \& Lutkenhaus, 2002).

Bacteria display a wide variety of cell shapes. Although some divisome proteins such as FtsZ are conserved among nearly all bacteria, other components of the cell division machinery diverge significantly to reflect the diversity of bacterial shapes. For example, the Min proteins are required for preventing $Z$ ring assembly at cell poles in Bacillus subtilis and $E$. coli but are missing from some cocci, such as Staphylococcus, Enterococcus, and Streptococcus (Zapun et al., 2008).

The mechanism of Fts $Z$ localization and the regulators that affect $Z$ ring assembly have been extensively studied in the rod-shaped laboratory workhorses $E$. coli and $B$. subtilis. In this study, we report for the first time that $\mathrm{Ga} 5 \mathrm{DH}$ plays a role in the cell growth and cell division of $S$. suis. In the absence of $\mathrm{Ga} 5 \mathrm{DH}$, cells exhibited a reduced growth rate and plump sausage-like shape with non-constricted septa joining the progeny. We found that the cells lacking $\mathrm{Ga} 5 \mathrm{DH}$ display aberrant formation of FtsZ rings. Furthermore, protein interaction studies revealed that $\mathrm{Ga} 5 \mathrm{DH}$ is capable of binding to FtsZ in vitro. These results suggest that $\mathrm{Ga} 5 \mathrm{DH}$ is involved in maintaining correct cell shape and in cell division.

\section{RESULTS}

\section{Construction of $\Delta \mathrm{ga} 5 \mathrm{dh}$ in S. suis $05 \mathrm{ZYH} 33$}

SS2 05ZYH33 was isolated from patients with streptococcal toxic shock syndrome (STSS) in Sichuan Province, China (Tang et al., 2006). To further investigate the function of $\mathrm{Ga5DH}$ in the bacterial physiology of SS2, we constructed a homologous suicide plasmid, pUC::ga5dh, with a $s p c R$ cassette. The suicide plasmid was then transformed into S. suis, and positive transformants were screened on THY agar plates with the selective antibiotic spectinomycin. Successful construction of the $\Delta g a 5 d h$ strain was confirmed by multiplex-PCR analysis. In the $\Delta g a 5 d h$ mutant, the entire ga5dh gene was replaced with a spectinomycin cassette (Fig. 1A). Western blot assays confirmed the absence of $\mathrm{Ga5DH}$ in the deletion mutant and the return of $\mathrm{Ga5DH}$ expression in the complemented strain (Fig. 1B).

\section{Growth phenotype of the $\Delta g a 5 d h$ mutant}

We first characterized the growth kinetics of the mutant strain. The wild type (WT) and $\Delta$ ga $5 d h$ strains were grown overnight and then inoculated into fresh nutrient-rich THY medium at $37^{\circ} \mathrm{C}$. A reduced growth rate was observed for the Ga5DH-deficient cells compared to the WT strain, indicating that Ga5DH is critical for $S$. suis growth (Fig. 2A). Indeed, complementation of ga5dh (Cga5dh) partially restored the growth defect. It is noteworthy that in THY medium, where gluconate is not utilized as the main carbon source, the $\Delta g a 5 d h$ growth defect phenotype was still dramatic.

We further examined the cell growth of the WT, deletion mutant, and complemented strains in THY medium with extra carbon source by supplementation with $2 \%(w / v)$ gluconate sodium (Fig. 2B). Similar growth profiles to those obtained in THY medium were recorded. Based on these observations, we believe it is unlikely that the impaired cell growth in $\Delta g a 5 d h$ was due to an energy deficiency or exhaustion. Therefore, we hypothesized that Ga5DH might function to affect $S$. suis cell division, in addition to its traditional role as a gluconate metabolic enzyme.

\section{Deletion of the ga5dh gene affects cell morphology and division}

Scanning electron microscopy (SEM) was employed to study the morphology and division pattern of the WT and mutant $S$. suis strains. WT cells grew as diplococci or short chains showing a characteristic ellipsoid shape and normal division pattern. The $\Delta g a 5 d h$ strain, however, developed into plump, sausage-shaped cells that were less ovoid and significantly longer than the WT cells. In addition, multiple indentations appeared on the cell surface of the $\Delta g a 5 d h$ strain (Fig. $3 \mathrm{~A}$ ). $A$ quantitative analysis of the cell length was further performed to analyze the morphological phenotypes of the ga5dh deletion mutant (Fig. 3B and $3 \mathrm{C}$ ). Single membranestained cells were randomly selected, and their longitudinal length was measured. The statistics of cell length for the three $S$. suis strains (WT, $\Delta g a 5 d h$, and complemented) all revealed a typical Gaussian distribution pattern. Nevertheless, the WT and the complemented bacteria concentrate mainly in a length interval of 1.0-1.7 $\mu \mathrm{m}$, whereas the majority of the $\Delta g a 5 d h$ cells were distributed in a region with a cell length of $1.5-2.3 \mu \mathrm{m}$ (Fig. 3B). On average, the calculated cell length along the long axis in the deletion strain was $1.95 \mu \mathrm{m}(n=200)$, compared to $1.42 \mu \mathrm{m}$ in the WT cells $(n=200)$ and $1.55 \mu \mathrm{m}$ in the Ga5DH-complemented cells. 
A

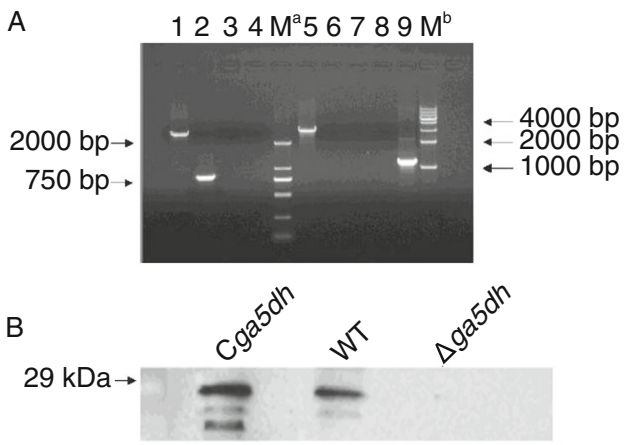

Figure 1. Construction and confirmation of the $\Delta g a 5 d h$ mutant $S$. suis and its complemented strain by multiplex PCR and Western blot analyses. (A) Genomic DNA extracts from the WT or the deletion mutant were used as templates: WT strain (lanes 1-4) and $\Delta$ ga5dh mutant (lanes 5-9). Two different DNA ladder markers, which are marked as $M^{a}$ and $M^{b}$ respectively, were used and labeled. The primer pairs and the theoretical size (bp) of the indicated PCR product are as follows: lanes 1: LU/RD, 2892 bp; 2: ga5dh-F/ga5dh-R, 835 bp; 3: ga5dh-F/spc-R, (-); 4: spc-F/ga5dh-R, (-); 5: LU/RD, 3209 bp; 6: ga5dh-F/ga5dh-R, (-); 7: ga5dh-F/spc-R, (-); 8: spc-F/ga5dh-R, (-); and 9: spc-F/spc-R, 1130 bp. (B) Western blot assay characterizing the protein expression of $\mathrm{Ga} 5 \mathrm{DH}$. The indicated S. suis cells were probed with $\mathrm{Ga} 5 \mathrm{DH}$ polyclonal antibodies, and the expression profiles are shown.

Cell width in $\Delta g a 5 d h$ cells was also slightly greater than that of the WT cells (Fig. 3C).

Additionally, we used transmission electron microscopy (TEM) and FM4-64 (a styryl membrane-specific dye) staining to gain an exact view of the cell shape and cell septa of both the WT and the mutant cells in details. In contrast to the WT cells with their typical ovoid shape, $\Delta$ ga $5 d h$ cells were enlarged, and their cell poles were round (Fig. 4A and 4B). Further, by staining with FM4-64, severe defects in morphology were observed in the $\Delta g a 5 d h$ cells. For example, the mutant cells were significantly longer and presented multiple aberrant septa. Cell constriction was also impaired, indicating that the septa might have no or abnormal functions. In addition, the $\Delta g a 5 d h$ cells exhibited severe division defects, including abnormal septum position (Fig. 4A) and asymmetrical divisions (Fig. 4B). For the WT strain, however, the dividing cells displayed the normal tight coordination of septum formation and constriction, showing characteristics of a symmetrical division. As expected, complementation of the ga5dh gene largely restored a normal celldividing phenotype in S. suis. These data indicate that Ga5DH is involved in $S$. suis cell division and suggest that $\mathrm{Ga} 5 \mathrm{DH}$ might play a role in septum constriction.

\section{Distribution of Ga5DH at mid-cell sites}

We further tested the subcellular localization of $\mathrm{Ga5DH}$ in exponentially growing cells by fluorescence microscopy using polyclonal antibodies directed against $\mathrm{Ga} 5 \mathrm{DH}$. As expected, no signal was detected in the $\Delta g a 5 d h$ cells (Fig. 5). However, the Ga5DH protein in both the WT and the complemented cells was mainly distributed as bands at the cell division septum (Fig. 5).

\section{Ga5DH interacts with FtsZ in vitro}

The subcellular localization of $\mathrm{Ga5DH}$ at the cell division septum implied a potential interaction of $\mathrm{Ga5DH}$ with FtsZ, an important cell division initiation factor that also localizes at this site. A variety of assays were performed to evaluate the physical interactions between the Ga5DH and Fts $\mathrm{Z}$ proteins of $S$. suis in vitro. First, FtsZ- or BSA-coated microtiter enzyme-linked immunosorbent assay (ELISA) plates were incubated with varying concentrations of Ga5DH. Following washing, the bound $\mathrm{Ga} 5 \mathrm{DH}$ protein was immunolabeled and quantified by ELISAs. As shown in Fig. 6A, Ga5DH bound to the FtsZ-coated wells but not to the wells coated with BSA.
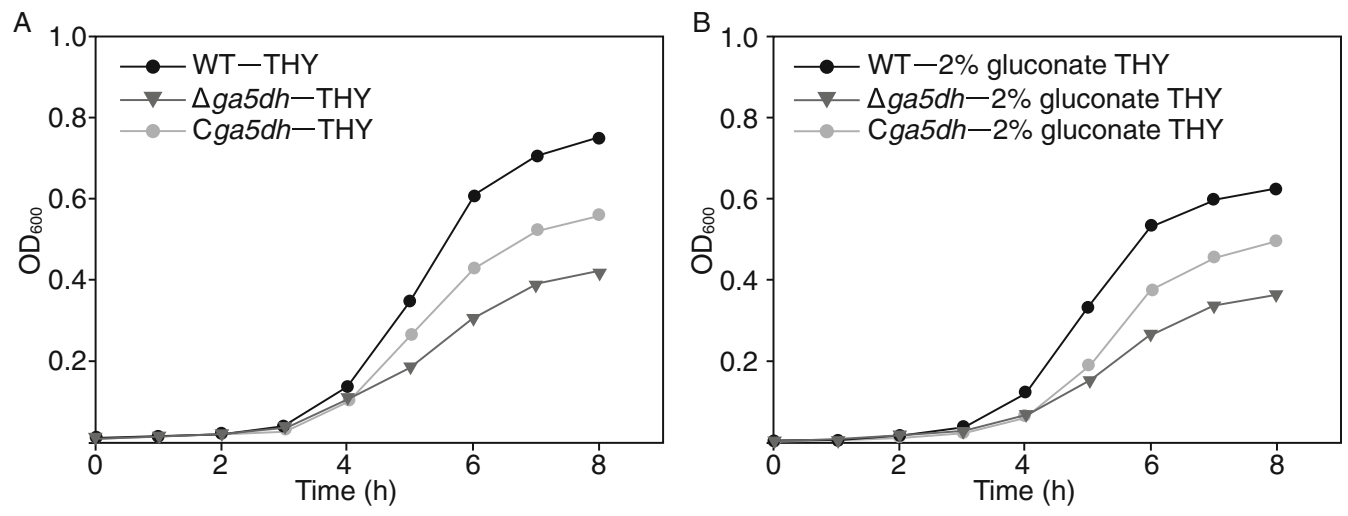

Figure 2. Ga5DH is required for $S$. suis growth. Comparative analyses of growth curves from three $S$. suis strains (WT, the $\Delta$ ga5dh mutant, and the complemented strain Cga5dh). The strains were grown in THY liquid medium (A) or in THY liquid medium supplemented with $2 \%(w / v)$ gluconate $(B)$. Each point represents the mean $\pm S D D_{600}$ value from triplicate experiments. 
A

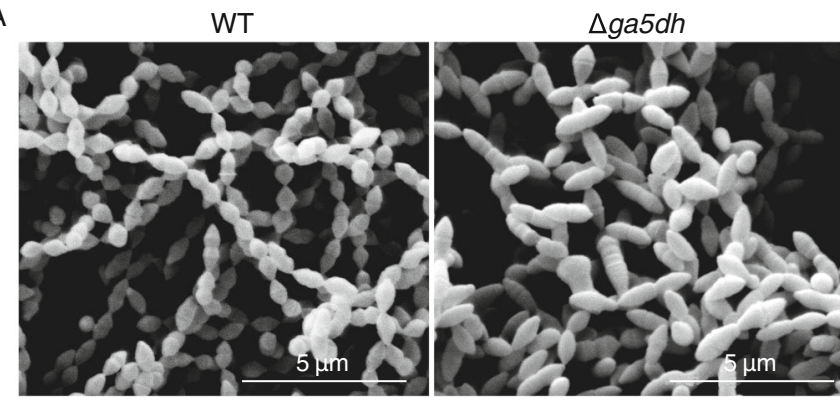

B

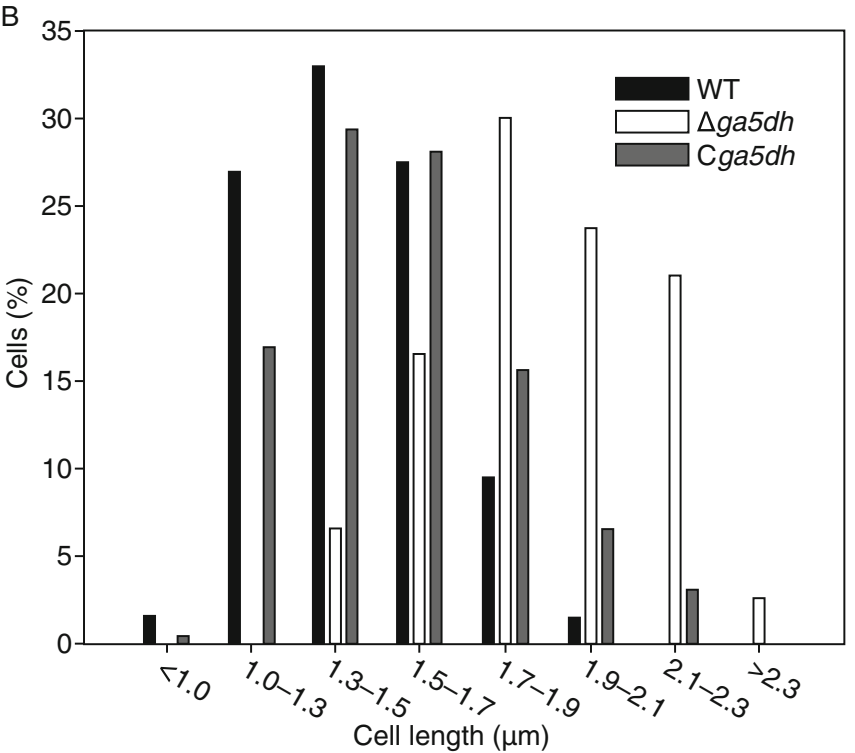

C
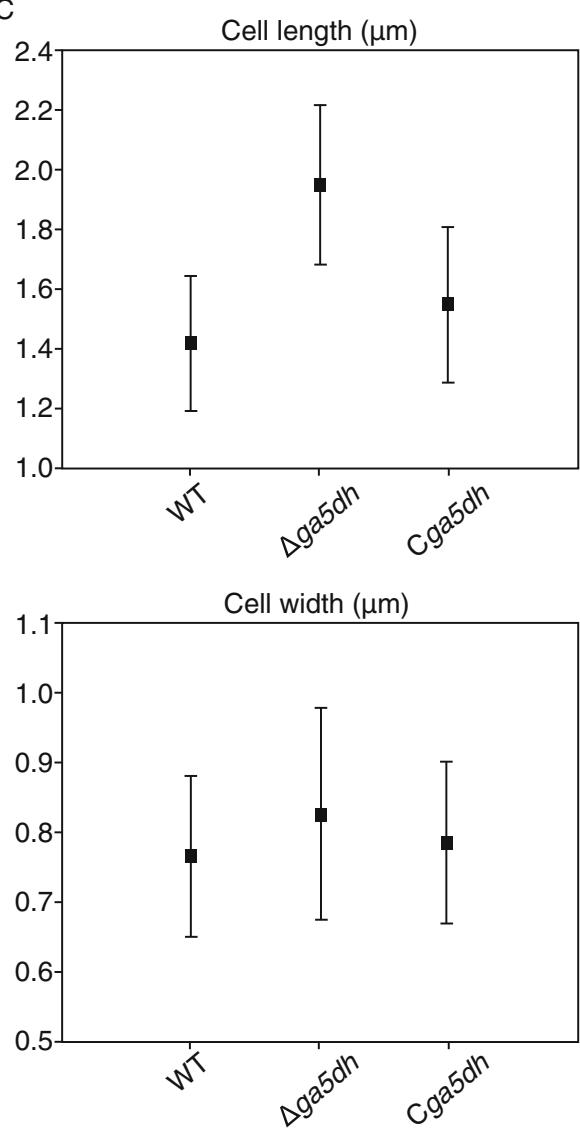

Figure 3. Micrographs of WT, $\Delta$ ga5dh mutant cells, and Cga5dh cells. (A) Scanning electron micrographs of the WT and the $\Delta$ ga5dh mutant strains. (B) Histogram statistics of the WT ( $n=200$, black), $\Delta$ ga5dh ( $n=200$, white), and Cga5dh ( $n=200$, gray) cell lengths after growth in THY medium. To determine the cell length, we measured the longitudinal length of single membrane-stained cells. $\Delta$ ga5dh cells were significantly longer than WT cells. (C) Cell size parameters of the WT, $\Delta g a 5 d h$, and Cga5dh cells. The cell length of $\Delta$ ga5dh cells was significantly different from that of the WT strain $(P<0.01$; two-tailed $t$-test).

Next, GST pull-down assays were performed to further confirm the interaction between Ga5DH and FtsZ. FtsZ was $\mathrm{N}$-terminally fused to GST, expressed in $E$. coli, and purified to homogeneity (Fig. 6B). To determine whether Ga5DH and FtsZ could form a stable complex, equimolar amounts of GST-FtsZ or GST were mixed with E. coli extracts containing Ga5DH and loaded onto a column packed with glutathione resin. Following washing, the bound proteins were eluted with glutathione. Bound Ga5DH, which was identified by Western blotting, was detected only for GST-FtsZ but not for the control protein of purified GST (Fig. 6C). Therefore, we provide solid evidence that Ga5DH directly interacts with FtsZ.

\section{Deletion of Ga5DH results in $\mathrm{Z}$ ring delocalization}

The in vitro observation of a direct Ga5DH/FtsZ interaction urged us to further analyze the effect of Ga5DH deletion on FtsZ localization in vivo. Immunofluorescence assays of septal FtsZ ring morphology in the WT and $\Delta$ ga5dh cells were performed using a polyclonal antibody against FtsZ. As expected, the majority of WT cells displayed FtsZ protein that was regularly distributed as a line at mid-cell (Fig. 7). The FtsZ localization in the $\Delta$ ga5dh cells, however, was dramatically altered compared to that in the WT cells. For the Ga5DH deletion mutant, FtsZ was present in aberrant singlets or doublets, distributed along the length of the cell. Moreover, in the absence of Ga5DH, some FtsZ failed to localize to the potential division sites. As expected, complementation of the ga5dh gene faithfully restored a normal FtsZ localization pattern (Fig. 7). This suggests that in addition to its enzymatic activity, Ga5DH may play a physical role in $S$. suis cell division for $Z$ ring localization.

\section{DISCUSSION}

Tight regulation of the cell wall synthesis and degradation machinery is required to maintain the proper shape and size of bacterial cells. Septal cell wall formation during division must be coordinated with other processes of cell division, such as 


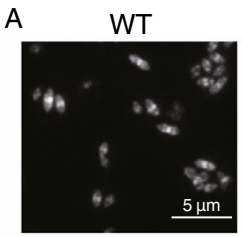

B

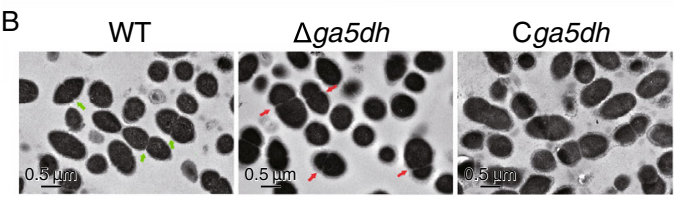

Figure 4. Micrographs of the WT, $\Delta$ ga5dh, and Cga5dh strains. (A) FM4-64 staining; scale bar, $5 \mu \mathrm{m}$. (B) Transmission electron micrographs; scale bar, $0.5 \mu \mathrm{m}$. Green arrows highlight the correct septum placement in WT cells. Red arrows mark the aberrant septum placement in $\Delta g a 5 d h$ cells. Cell morphology of the WT strain revealed the characteristic ellipsoid shape and normal division pattern of $S$. suis, with correct septum placement and symmetric daughter cells. The mutant cells displayed an increased length and multiple aberrant septa with incorrect placement.

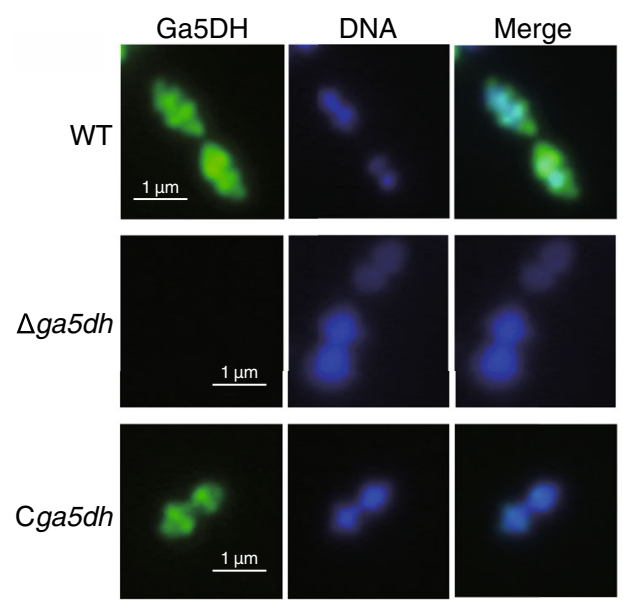

Figure 5. Subcellular localization of Ga5DH. DNA was visualized with DAPI (blue). Ga5DH was visualized using an anti-Ga5DH polyclonal antibody and an anti-mouse lgG secondary antibody coupled to Alexa Fluor 488 (green). The merged pictures show the overlay of $\mathrm{Ga} 5 \mathrm{DH}$ and DAPI staining. Scale bar, $1 \mu \mathrm{m}$.

membrane invagination, DNA replication, and chromosome segregation. To avoid a catastrophic breakage of the chromosome during division, cell separation must occur exactly at the middle of the cell to ensure the generation of two equal daughter cells. It is known that the initiation of cell division requires the proper location of the $\mathrm{Z}$ ring at mid-cell. Here, our data indicate that $\mathrm{Ga} 5 \mathrm{DH}$, a gluconate metabolic enzyme, has an important role in $S$. suis cell division.

The deletion of ga5dh led to aberrant cell shape and division. Compared to the WT cells, the deletion mutant exhibited increased length, with non-constricted septa, reminiscent of the phenotypes exhibited by pneumococcus stkP-KD-TMH mutant cells (Fleurie et al., 2012). Fleurie et al. confirmed that the misplacement of StkP abolishes its function and affects cell division. The same cell morphology was also observed in pneumococcus cells with PBP $2 \mathrm{~b}^{28}$ (a mutant PBP2b sequence obtained from the Streptococcus pneumoniae Cba-28 clinical strain) proteins, which display a rod-like shape (Albarracin Orio et al., 2011). TEM revealed that the rod-shaped cells exhibit multiple septa, and it was proposed that the direct interaction of PBP2 $b^{28}$ with FtsZ leads to the mislocalization of FtsZ and to an aberrant cellular morphology. Here, we describe plump, sausage-shaped cells and an abnormal cell division pattern caused by the deletion of Ga5DH. Moreover, we confirmed that Ga5DH localized mainly at the septum and directly interacted with the essential cell division initiation factor FtsZ. In addition, the deletion of $\mathrm{Ga5DH}$ altered the normal mid-cell localization of FtsZ. These observations indicate a role of $\mathrm{Ga} 5 \mathrm{DH}$ in the FtsZ localization at the septal site, though it remains to be investigated whether $\mathrm{Ga} 5 \mathrm{DH}$ has a direct or an indirect impact on $\mathrm{Fts} Z$ assembly and subsequent cell division.

The primary role of $\mathrm{Ga} 5 \mathrm{DH}$ is metabolic, catalyzing the inter-conversion of D-gluconate and 5-keto-D-gluconate. In previous studies, other metabolic proteins such as ManA and UgtP have been reported to affect cellular structures and cell division in B. subtilis (Elbaz \& Ben-Yehuda, 2010; Weart et al., 2007). In B. subtilis, nutrient availability has a dramatic effect on UDP-glucose accumulation. Under conditions in which UDP-glucose levels are high, UDP-glucose directly interacts with the diacylglycerol glucosyltransferase UgtP to inhibit FtsZ assembly and delay maturation of the $Z$ ring. It is possible that the metabolic enzyme Ga5DH operates as a sensor that synchronizes cell division with metabolite availability through its role in coordinating FtsZ localization.

\section{MATERIALS AND METHODS}

Bacterial strains, plasmids, and culture conditions

Strains and plasmids used in this study are listed in Table 1. Streptococcus suis strains were grown in Todd-Hewitt broth (THB) (Difco Laboratories, Detroit, MI, USA) supplemented with 2\% yeast extract (THY) or plated on THY agar at $37^{\circ} \mathrm{C}$ (Feng et al., 2007; Feng et al., 2008). THY medium and THY medium supplemented with $2 \%$ gluconate were used for growth curve measurements. Experiments were performed in triplicate.

E. coli strains $\mathrm{DH} 5 \alpha$ and BL21 were incubated in Luria-Bertani (LB) medium or plated on LB agar. When required, antibiotics were routinely added at the following concentrations: spectinomycin, $100 \mathrm{mg} / \mathrm{mL}$ for both $S$. suis and E. coli; erythromycin, $1 \mathrm{mg} / \mathrm{mL}$ for S. suis and $250 \mathrm{mg} / \mathrm{mL}$ for E. coli. $50 \mathrm{mg} / \mathrm{mL}$ of ampicillin was always present to screen the transformants of $E$. coli.

Cloning, mutagenesis, and genetic complementation

To delete ga5dh, the two flanking sequences of ga5dh were amplified from the chromosomal DNA of S. suis 05ZYH33 (Feng et al., 2008; 


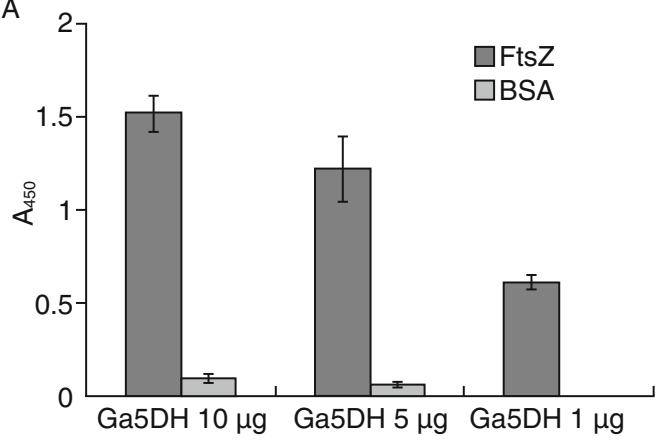

B

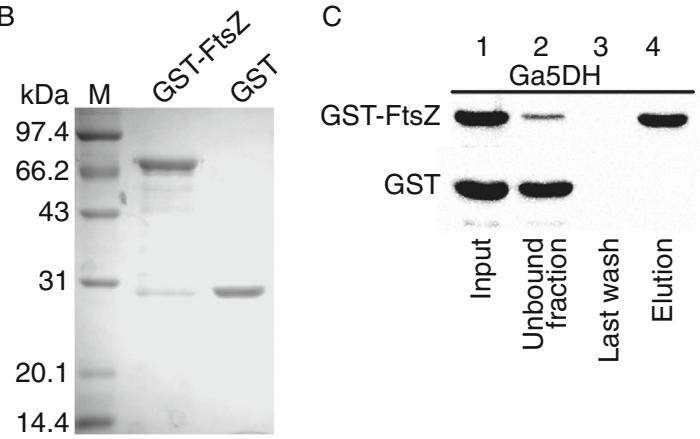

Figure 6. Ga5DH interacts with FtsZ in vitro. (A) Solid-phase binding assay for FtsZ and Ga5DH. Wells of a microtiter plate were coated with FtsZ or BSA and incubated with various concentrations of $\mathrm{Ga} 5 \mathrm{DH}$ protein as indicated. The bound $\mathrm{Ga5DH}$ protein was immunodetected with $\mathrm{Ga} 5 \mathrm{DH}$ polyclonal antibodies by ELISA. (B) Inputs of GST-FtsZ and GST in the GST pull-down assay detected by SDS-PAGE. (C) GST pulldown assay characterizing the interaction between FtsZ and Ga5DH. Lane 1: the input His-tagged Ga5DH in the pull-down assay. Lane 2: the unbound fraction of $\mathrm{Ga} 5 \mathrm{DH}$. Lane 3: the last wash of the unbound fraction of $\mathrm{Ga5DH}$. Lane 4: the eluted protein fraction using $20 \mathrm{mmol} / \mathrm{L}$ glutathione. All samples were detected by Western blotting using an anti-His monoclonal antibody.

Li et al., 2008; Li et al., 2011). All primers used in this study are listed in Table 2. For cloning ga5dh homologous regions, two pairs of specific primers (LA-P1/LA-P2 and RA-P1/RA-P2) carrying EcoRI/BamHI and $P$ stl/HindIII restriction enzyme sites were used, respectively. After digestion with the corresponding restriction enzymes, the DNA fragments were cloned into a pUC18 vector to generate the recombinant plasmid pUC::ga5dhLR. Then, the $s p c R$ gene cassette was inserted into BamHI/Pstl digested plasmid pUC::ga5dhLR to generate the ga5dh knockout plasmid pUC::ga5dh. To obtain the isogenic mutant $\triangle$ ga5dh, S. suis $05 \mathrm{ZYH} 33$ was transformed by electroporation of the resulting plasmid pUC::ga5dh following previously described procedures (Feng et al., 2008; Li et al., 2008).

To construct the complement strain, a DNA fragment encoding ga5dh and its promoter region were generated by PCR using specific primers (Cga5dh-F/Cga5dh-R) and inserted into the digested E. coli-S. suis shuttle vector pVA838 (Macrina et al., 1982). And then the resulting plasmid pVA::ga5dh was transformed by electroporation into the $\Delta g a 5 d h$ mutant. Transformants were

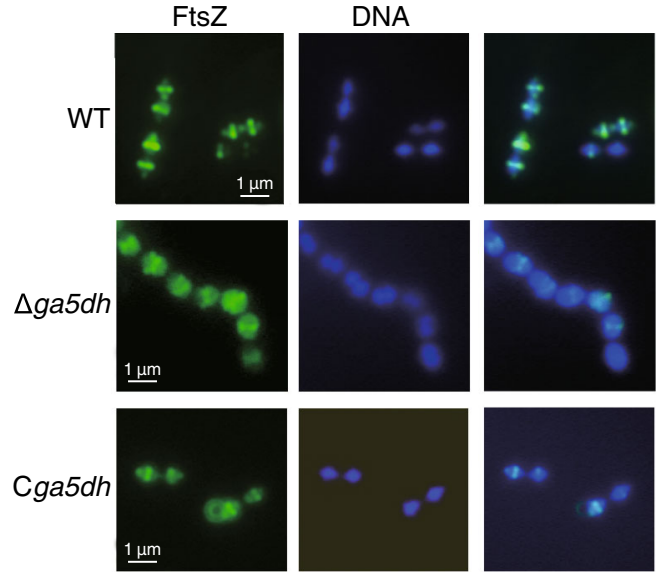

Figure 7. Delocalization of FtsZ rings in $S$. suis cells lacking $\mathrm{Ga} 5 \mathrm{DH}$. The morphology of $\mathrm{FtsZ}$ was detected by immunolabeling with anti-FtsZ polyclonal antibodies and an anti-mouse IgG secondary antibody coupled to Alexa Fluor 488 (green). DNA was visualized with DAPI (blue). The merged pictures show the overlay of FtsZ and DAPI staining. Scale bar $=1 \mu \mathrm{m}$.

screened on THY plates with selection for spectinomycin and erythromycin resistance.

\section{Overexpression and purification of FtsZ and Ga5DH}

The fts $Z$ gene was amplified by PCR using the chromosomal DNA of $S$. suis $05 Z \mathrm{YH} 33$ as template and specific primers described in Table 2. The ftsZ PCR product was digested by enzymes Ndel and Xhol and then inserted into the digested $\mathrm{pET} 30$ a vector to generate the recombinant plasmid pET30-ftsZ. For expression of FtsZ with a fused GST tag, ftsZ gene was cloned into the pGEX-6P-1 vector via the BamHI and EcoRI sites. For expression of His-tagged Ga5DH, the ga5dh PCR product was inserted into the pET28b vector via the Ndel and Xhol restriction sites (Zhang et al., 2009). The resulting recombinant plasmid was termed pET28b-ga5dh. In each case, the recombinant proteins were over-expressed in E. coli BL21 (DE3) in Luria Broth medium.

Cells from overnight liquid were grown to an $\mathrm{OD}_{600}$ of 0.4 and then induced by addition of $0.5 \mathrm{mmol} / \mathrm{L}$ IPTG. The cells were grown for an additional $3 \mathrm{~h}$ at $37^{\circ} \mathrm{C}$. The cells were harvested by centrifugation and resuspended in a buffer consisting of $20 \mathrm{mmol} / \mathrm{L}$ Tris- $\mathrm{HCl}$ $\mathrm{pH} 8.0$ and $50 \mathrm{mmol} / \mathrm{L} \mathrm{NaCl}$ and sonicated. After centrifugation, the supernatant was applied to a $5-\mathrm{mL}$ column of HisTrap FF resin or of Glutathione Sepharose FF resin (GE Healthcare). Following extensive washing with the binding buffer $(20 \mathrm{mmol} / \mathrm{L}$ Tris- $\mathrm{HCl} \mathrm{pH} 8.0$, $50 \mathrm{mmol} / \mathrm{L} \mathrm{NaCl})$, samples were eluted with either buffer $A(20 \mathrm{mmol} / \mathrm{L}$ Tris- $\mathrm{HCl} \mathrm{pH} \mathrm{8.0,50} \mathrm{mmol/L} \mathrm{NaCl,} 300 \mathrm{mmol} / \mathrm{L}$ imidazole) for Histagged proteins or buffer $\mathrm{B}(20 \mathrm{mmol} / \mathrm{L}$ Tris- $\mathrm{HCl} \mathrm{pH} 8.0,50 \mathrm{mmol} / \mathrm{L}$ $\mathrm{NaCl}, 20 \mathrm{mmol} / \mathrm{L}$ glutathione) for GST-tagged proteins. Peak fractions were pooled. Proteins were further purified by gel filtration chromatography using a Superdex-200 10/300 GL column (GE Healthcare) with $20 \mathrm{mmol} / \mathrm{L}$ Tris- $\mathrm{HCl}$ and $50 \mathrm{mmol} / \mathrm{L} \mathrm{NaCl}, \mathrm{pH} 8.0$ as running buffer and then stored at $-80^{\circ} \mathrm{C}$. The His-tagged FtsZ and $\mathrm{Ga} 5 \mathrm{DH}$ were individually used to immunize mice to produce polyclonal antibodies. 
Table 1. Bacterial strains and plasmids used in this study

\begin{tabular}{|c|c|c|}
\hline $\begin{array}{l}\text { Strains/ } \\
\text { plasmids }\end{array}$ & Characteristics & Origins \\
\hline \multicolumn{3}{|c|}{ Bacterial strains } \\
\hline 05ZYH33 & $\begin{array}{l}\text { A virulent Chinese isolate } \\
\text { of } S \text {. suis serotype } 2\end{array}$ & $\begin{array}{l}\text { Feng et al. } \\
\text { (2008); Tang } \\
\text { et al. (2006) }\end{array}$ \\
\hline $\begin{array}{r}05 Z \mathrm{YH} 33 \\
\Delta g a 5 d h\end{array}$ & $\begin{array}{l}\text { 05ZYH33 derivative with } \\
\text { the ga5dh gene replaced } \\
\text { by a spcR gene cassette }\end{array}$ & This work \\
\hline Cga5dh & $\begin{array}{l}\text { Complemented strain of } \\
05 Z Y H 33 \Delta \text { ga5dh }\end{array}$ & This work \\
\hline $\mathrm{DH} 5 \alpha$ & An E. coli cloning host & Lab stock \\
\hline BL21 & An E. coli expression host & Lab stock \\
\hline \multicolumn{3}{|l|}{ Plasmids } \\
\hline pUC-18 & Cloning vector & TaKaRa \\
\hline pET28b & $\begin{array}{l}\text { His-tag fusion expression } \\
\text { vector }\end{array}$ & Novagen \\
\hline pET30a & $\begin{array}{l}\text { His-tag fusion expression } \\
\text { vector }\end{array}$ & Novagen \\
\hline $\begin{array}{l}\text { pGEX- } \\
6 \text { P-1 }\end{array}$ & $\begin{array}{l}\text { GST-tag fusion expression } \\
\text { vector }\end{array}$ & Novagen \\
\hline pVA-838 & $\begin{array}{l}\text { E. coli - S. suis shuttle } \\
\text { vector }\end{array}$ & Lab stock \\
\hline
\end{tabular}

Solid phase binding assay

Five hundred micrograms of purified FtsZ or BSA were coated in a buffer containing $14 \mathrm{mmol} / \mathrm{L} \mathrm{Na}_{2} \mathrm{CO}_{3}$ and $36 \mathrm{mmol} / \mathrm{L} \mathrm{NaHCO}$ overnight at $4^{\circ} \mathrm{C}$ onto the enzyme-linked immunosorbent assay plates. After adsorption, the wells were washed three times with phosphate-buffer saline containing $0.5 \%$ Tween-20 (PBST) to remove the excess unbound proteins. Nonspecific binding sites of the wells were blocked with $10 \%$ goat serum in PBS for $2 \mathrm{~h}$ at $37^{\circ} \mathrm{C}$. This was followed by incubation for $2 \mathrm{~h}$ at $37^{\circ} \mathrm{C}$ with different concentrations of Ga5DH protein in PBS. The plates were then rinsed with PBST three times. Finally, the interaction of Ga5DH with FtsZ was detected with anti-Ga5DH antibodies by an enzyme-linked immunosorbent assay. The anti-Ga5DH antibodies used for the immunosorbent assay were pretreated with immobilized His-tagged enolase protein (Lu et al., 2012) to fish out anti-His antibodies. Wells coated with BSA served as negative controls. All assays were performed in triplicate.

\section{GST pull down assay}

The pull down assay was performed with the purified GST-FtsZ or GST proteins by utilizing a modified method (Dziedzic et al., 2010). In brief, equal-molar amounts of GST-FtsZ or GST in PBS were mixed and incubated at $4^{\circ} \mathrm{C}$ with $E$. coli lysates containing His-tagged $\mathrm{Ga} 5 \mathrm{DH}$ for about $4 \mathrm{~h}$. Then the mixtures were allowed to bind to glutathione-Sepharose. The slurries were washed thoroughly with PBS. The bound proteins were eluted with glutathione. The samples were boiled in SDS-PAGE loading buffer for $10 \mathrm{~min}$. The separated proteins were subjected to SDS-PAGE and immunoblotted. Samples were probed with an anti-His monoclonal antibody (MBL).

\section{Immunofluorescence microscopy}

Immunofluorescence was performed as previously described with small modifications (Foulquier et al., 2011). Cells from overnight culture were diluted 100 -fold into $3 \mathrm{~mL}$ fresh THY medium and grown

Table 2. Primers used in this study

\begin{tabular}{|c|c|c|}
\hline Primers & Sequence $\left(5^{\prime}-3^{\prime}\right)$ & Function \\
\hline LU & TTCCGGCTGTTTACCAATGT & \multirow[t]{2}{*}{ PCR detection } \\
\hline $\mathrm{RD}$ & CTAAAGGGAAAAATTGCTTTG & \\
\hline LA-P1 (EcoRI) & CCGGAATTCATAGCTTCCAAACTAGACTGG & \multirow[t]{2}{*}{ Mutant strain construction } \\
\hline LA-P2 (BamHI) & CGCGGATCCATAAGAAGGGAAAAAACGATG & \\
\hline RA-P1 (Pstl) & AACTGCAGATTCGTAACTCCСTTGTATTT & \multirow[t]{2}{*}{ Mutant strain construction } \\
\hline RA-P2 (HindIII) & CCCAAGCTTTTGTTTTCATGTACACTTTTG & \\
\hline Cga5dh-F (BamHI) & CGCGGATCCCTGATATGACTATATAAAATG & \multirow[t]{2}{*}{ ga5dh cloning and complementation } \\
\hline Cga5dh-R (Sall) & GCGTCGACTTACGCTTCCGGCTGTTTA & \\
\hline spc-F (BamHI) & GCAGGATCCGTTCGTGAATACATGTTATA & \multirow[t]{2}{*}{$s p c$ cloning and PCR detection } \\
\hline $\operatorname{spc}-\mathrm{R}(P s t l)$ & GGCTGCAGGTTTTCTAAAATCTGAT & \\
\hline ftsZ-F (Ndel) & GGAATTCCATATGGCATTTTCATTTGAAGCA & \multirow[t]{2}{*}{ ftsZ cloning into $\mathrm{pET} 30 \mathrm{a}$ and protein expression } \\
\hline ftsZ-R (Xhol) & CCGCTCGAGGCGATTACGGAAGAATGG & \\
\hline ftsZ-GST-F(BamHI) & CGCGGATCCATGGCATTTTCATTTGAAGCA & \multirow[t]{2}{*}{ ftsZ cloning into pGEX-6P-1 and protein expression } \\
\hline ftsZ-GST-R(EcoRI) & CCGGAATTCTTAGCGATTACGGAAGAATGG & \\
\hline ga5dh-F (Ndel) & GGAATTCCATATGAATCAGCAATTTTC & \multirow{2}{*}{$\begin{array}{l}\text { ga5dh cloning in pET28b and protein } \\
\text { expression (Zhang et al., 2009) }\end{array}$} \\
\hline ga5dh-R (Xhol) & CCGCTCGAGTTACGCTTCCGGCTGTTT & \\
\hline
\end{tabular}

The underlined sequences represent the restriction sites. 
at $37^{\circ} \mathrm{C}$ to exponential phase. The cells $(1 \mathrm{~mL})$ were then harvested and resuspended in $10 \mathrm{~mL}$ of ice-cold $80 \%$ methanol and incubated for $1 \mathrm{~h}$ at room temperature. The suspension was then concentrated, resuspended in $200 \mu \mathrm{L}$ of freshly prepared $16 \%$ formaldehyde and incubated for $5 \mathrm{~min}$ at room temperature. Then samples were centrifuged and washed once with $1 \mathrm{~mL}$ of ice-cold $80 \%$ methanol. After centrifugation, cells were permeabilized at $37^{\circ} \mathrm{C}$ for $10 \mathrm{~min}$ in $20 \mathrm{mmol} / \mathrm{L}$ sodium phosphate buffer $\mathrm{pH} 6.2,50 \mathrm{mmol} / \mathrm{L}$ sucrose, $500 \mathrm{mg} / \mathrm{mL}$ lysozyme. Cells were then washed with PBST, saturated with $200 \mu \mathrm{L}$ of PBST-10\% goat serum containing anti-Ga5DH or anti-FtsZ antibodies (dilution 1/100) and incubated overnight at $4^{\circ} \mathrm{C}$. The samples were washed twice with PBST and incubated with a 1:300 dilution of the secondary goat anti-mouse antibody coupled with Alexa Fluor 488 (Santa Cruz) in the dark. DNA was visualized by treatment with the DNA fluorescent stain DAPI. For FM4-64 staining, all cells were harvested and incubated in $5 \mu \mathrm{mol} / \mathrm{L}$ FM4-64 in dark for $20 \mathrm{~min}$. The excess dye was washed with THY medium. Finally, cells were mounted directly onto microscope slides covered with a thin film of $1.2 \%$ agar in water. Fluorescent images were acquired by laser scanning confocal microscope (Leica TCS SP2).

\section{Transmission electron microscopy}

All samples were harvested at an $\mathrm{OD}_{600}$ of 0.8 and fixed with $2.5 \%$ glutaraldehyde $(1 \mathrm{~mL})$ followed by washing with PBS. The cells were treated with $1 \%$ osmium tetroxide for $2 \mathrm{~h}$ in dark. Then the subsequent dehydration steps with ethanol were carried out as follows: $50 \%$ for $15 \mathrm{~min}, 70 \%$ for $15 \mathrm{~min}, 95 \%$ for $15 \mathrm{~min}, 100 \%$ for $20 \mathrm{~min}$. The samples were embedded in Spurr's plastic and sectioned. Cell morphology was then visualized using a JEM-1400 (JEDL) transmission electron microscope.

\section{Scanning electron microscopy}

All samples were grown in THY broth and harvested at an $\mathrm{OD}_{600}$ of 0.8. Cells were spotted onto polylysine coverslips followed by washing with PBS. The cells were fixed in $0.18 \mathrm{~mol} / \mathrm{L}$ cacodylate buffer ( $\mathrm{pH} 7.6$ ) containing $2 \%$ glutaraldehyde. Then the subsequent dehydration steps with ethanol were carried out, passage in HMDS (1,1,1,3,3,3-hexamethyldisila zane) and finally air dried. The dried samples were covered with a $10 \mathrm{~nm}$-thick gold/platinum layer. Samples were then observed with a Quanta200 (FEI) scanning electron microscope.

\section{ACKNOWLEDGEMENTS}

This work is supported by the HCJRG program (GJHZ1307). GFG is a leading principal investigator of the National Natural Science Foundation of China (NSFC) Innovative Research Group (Grant No. 81321063). We thank Jingnan Liang for the technical assistance.

\section{COMPLIANCE WITH ETHICS GUIDELINES}

Zhongyu Shi, Chunling Xuan, Huiming Han, Xia Cheng, Jundong Wang, Youjun Feng, Swaminath Srinivas, Guangwen Lu, and George F. Gao declare that they have no conflict of interest.

This article does not contain any studies with human or animal subjects performed by the any of the authors.

\section{OPEN ACCESS}

This article is distributed under the terms of the Creative Commons Attribution License which permits any use, distribution, and reproduction in any medium, provided the original author(s) and the source are credited.

\section{REFERENCES}

Addinall SG, Lutkenhaus J (1996) FtsZ-spirals and -arcs determine the shape of the invaginating septa in some mutants of Escherichia coli. Mol Microbiol 22:231-237

Albarracin Orio AG, Pinas GE, Cortes PR, Cian MB, Echenique J (2011) Compensatory evolution of $p b p$ mutations restores the fitness cost imposed by $\beta$-lactam resistance in Streptococcus pneumoniae. PLoS Pathog 7:e1002000

Bi EF, Lutkenhaus J (1991) FtsZ ring structure associated with division in Escherichia coli. Nature 354:161-164

Dziedzic R, Kiran M, Plocinski P, Ziolkiewicz M, Brzostek A, Moomey M, Vadrevu IS, Dziadek J, Madiraju M, Rajagopalan M (2010) Mycobacterium tuberculosis ClpX interacts with FtsZ and interferes with FtsZ assembly. PloS One 5:e11058

Elbaz M, Ben-Yehuda S (2010) The metabolic enzyme ManA reveals a link between cell wall integrity and chromosome morphology. PLoS Genet 6:e1001119

Feng Y, Zheng F, Pan X, Sun W, Wang C, Dong Y, Ju A, Ge J, Liu D, Liu C et al (2007) Existence and characterization of allelic variants of Sao, a newly identified surface protein from Streptococcus suis. FEMS Microbiol Lett 275:80-88

Feng Y, Li M, Zhang H, Zheng B, Han H, Wang C, Yan J, Tang J, Gao GF (2008) Functional definition and global regulation of Zur, a zinc uptake regulator in a Streptococcus suis serotype 2 strain causing streptococcal toxic shock syndrome. J Bacteriol 190:7567-7578

Feng Y, Shi X, Zhang H, Zhang S, Ma Y, Zheng B, Han H, Lan Q, Tang J, Cheng J et al (2009) Recurrence of human Streptococcus suis infections in 2007: three cases of meningitis and implications that heterogeneous $S$. suis 2 circulates in China. Zoonoses Public Health 56:506-514

Feng Y, Zhang H, Ma Y, Gao GF (2010) Uncovering newly emerging variants of Streptococcus suis, an important zoonotic agent. Trends Microbiol 18:124-131

Fleurie A, Cluzel C, Guiral S, Freton C, Galisson F, Zanella-Cleon I, Di Guilmi AM, Grangeasse C (2012) Mutational dissection of the S/T-kinase StkP reveals crucial roles in cell division of Streptococcus pneumoniae. Mol Microbiol 83:746-758

Foulquier E, Pompeo F, Bernadac A, Espinosa L, Galinier A (2011) The YvcK protein is required for morphogenesis via localization of PBP1 under gluconeogenic growth conditions in Bacillus subtilis. Mol Microbiol 80:309-318

Kay R, Cheng AF, Tse CY (1995) Streptococcus suis infection in Hong Kong. QJM 88:39-47

Li M, Wang C, Feng Y, Pan X, Cheng G, Wang J, Ge J, Zheng F, Cao $M$, Dong $Y$ et al (2008) SalK/SalR, a two-component signal transduction system, is essential for full virulence of highly invasive Streptococcus suis serotype 2. PLoS One 3:e2080

Li M, Shen X, Yan J, Han H, Zheng B, Liu D, Cheng H, Zhao Y, Rao $X$, Wang C (2011) Gl-type T4SS-mediated horizontal transfer of 
the $89 \mathrm{~K}$ pathogenicity island in epidemic Streptococcus suis serotype 2. Mol Microbiol 79:1670-1683

Lu Q, Lu H, Qi J, Lu G, Gao GF (2012) An octamer of enolase from Streptococcus suis. Protein Cell 3:769-780

Macrina FL, Tobian JA, Jones KR, Evans RP, Clewell DB (1982) A cloning vector able to replicate in Escherichia coli and Streptococcus sanguis. Gene 19:345-353

Mazokopakis EE, Kofteridis DP, Papadakis JA, Gikas AH, Samonis GJ (2005) First case report of Streptococcus suis septicaemia and meningitis from Greece. Eur J Neurol 12:487-489

Nghia HD, Hoa NT, le Linh D, Campbell J, Diep TS, Chau NV, Mai NT, Hien TT, Spratt B, Farrar J et al (2008) Human case of Streptococcus suis serotype 16 infection. Emerg Infect Dis 14:155-157

Pichoff S, Lutkenhaus J (2002) Unique and overlapping roles for ZipA and FtsA in septal ring assembly in Escherichia coli. EMBO J 21:685-693
Sweeney NJ, Laux DC, Cohen PS (1996) Escherichia coli F-18 and E. coli K-12 eda mutants do not colonize the streptomycin-treated mouse large intestine. Infect Immun 64:3504-3511

Tang J, Wang C, Feng Y, Yang W, Song H, Chen Z, Yu H, Pan X, Zhou X, Wang H et al (2006) Streptococcal toxic shock syndrome caused by Streptococcus suis serotype 2. PLoS Med 3:e151

Weart RB, Lee AH, Chien AC, Haeusser DP, Hill NS, Levin PA (2007) A metabolic sensor governing cell size in bacteria. Cell 130:335-347

Zapun A, Vernet T, Pinho MG (2008) The different shapes of cocci. FEMS Microbiol Rev 32:345-360

Zhang Q, Peng H, Gao F, Liu Y, Cheng H, Thompson J, Gao GF (2009) Structural insight into the catalytic mechanism of gluconate 5-dehydrogenase from Streptococcus suis: crystal structures of the substrate-free and quaternary complex enzymes. Protein Sci 18:294-303 